\title{
Development and Characterization of Nisin Nanoparticles as Potential Alternative for the Recurrent Vaginal Candidiasis Treatment
}

\author{
Letícia Coli Louvisse de Abreu, ${ }^{1}$ Valerio Todaro, ${ }^{1}$ Plinio Cunha Sathler, ${ }^{1}$ \\ Luiz Cláudio Rodrigues Pereira da Silva, ${ }^{1}$ Flávia Almada do Carmo, ${ }^{1}$ \\ Cleonice Marques Costa, ${ }^{1}$ Helena Keiko Toma, ${ }^{3}$ Helena Carla Castro, ${ }^{4}$ \\ Carlos Rangel Rodrigues, ${ }^{2}$ Valeria Pereira de Sousa, ${ }^{1}$ and Lucio Mendes Cabral ${ }^{1,5}$
}

Received 17 September 2015; accepted 1 January 2016; published online 25 January 2016

\begin{abstract}
The aim of this work was the development and characterization of nisin-loaded nanoparticles and the evaluation of its potential antifungal activity. Candidiasis is a fungal infection caused by Candida sp. considered as one of the major public health problem currently. The discovery of antifungal agents that present a reduced or null resistance of Candida sp. and the development of more efficient drug release mechanisms are necessary for the improvement of candidiasis treatment. Nisin, a bacteriocin commercially available for more than 50 years, exhibits antibacterial action in food products with potential antifungal activity. Among several alternatives used to modulate antifungal activity of bacteriocins, polymeric nanoparticles have received great attention due to an effective drug release control and reduction of therapeutic dose, besides the minimization of adverse effects by the preferential accumulation in specific tissues. The nisin nanoparticles were prepared by double emulsification and solvent evaporation methods. Nanoparticles were characterized by dynamic light scattering, zeta potential, Fourier transform infrared, X-ray diffraction, differential scanning calorimetry, and scanning electron microscopy. Antifungal activity was accessed by pour plate method and cell counting using Candida albicans strains. The in vitro release profile and in vitro permeation studies were performed using dialysis bag method and pig vaginal mucosa in Franz diffusion cell, respectively. The results revealed nisin nanoparticles $(300 \mathrm{~nm})$ with spherical shape and high loading efficiency $(93.88 \pm 3.26 \%)$. In vitro test results suggest a promising application of these nanosystems as a prophylactic agent in recurrent vulvovaginal candidiasis and other gynecological diseases.
\end{abstract}

KEYWORDS: bacteriocins; candidiasis; gynecological diseases; nanoparticles; nisin.

\section{INTRODUCTION}

Currently, among all mycoses, candidiasis caused by Candida albicans and aspergillosis caused by Aspergillus fumigatus are considered the greatest public health problems (1). Candida spp. possess more than 200 species, of which some are found on microbial flora and only $10 \%$ can cause human

\footnotetext{
${ }^{1}$ Universidade Federal do Rio de Janeiro, LabTIF, Faculdade de Farmácia, Ilha do Fundão, CEP 21941-902, Rio de Janeiro, RJ, Brazil.

${ }^{2}$ Universidade Federal do Rio de Janeiro, ModMolQSAR, Faculdade de Farmácia, Ilha do Fundão, CEP 21941-902, Rio de Janeiro, RJ, Brazil.

${ }^{3}$ Universidade Federal do Rio de Janeiro, LACMAC, Faculdade de Farmácia, Ilha do Fundão, CEP 21941-902, Rio de Janeiro, RJ, Brazil.

${ }^{4}$ Universidade Federal Fluminense, LaBIEMol, Instituto de Biologia, Centro, CEP 24210-130, Niterói, RJ, Brazil.

${ }^{5}$ To whom correspondence should be addressed. (e-mail: lmcabral@pharma.ufrj.br; lmcabral2@yahoo.com.br)
}

diseases, especially in immunocompromised patients, such as those undergoing chemotherapy, under treatment with broad-spectrum antibiotic and individuals infected by HIV $(2,3)$.

Candidiasis occurs as an acute or chronic disease and even superficial or deep. The pathological processes include inflammation, purulent process, and granulomatous response. Some of the most affected areas are the oropharyngeal and vaginal mucosa, skin and appendages, bronchi, lungs, and gastrointestinal tract $(2,4)$. Eventually, some antifungal drugs are not effective for some fungi species, particularly against C. albicans. Failure response to antifungal therapy may be related to the intrinsic of resistance strains or acquired during the treatment $(3,5)$.

One of the current challenges is the development of an antifungal agent that presents a reduced or null resistance to Candida, or even the creation of a controlled release system able to increase the therapeutic efficacy of antifungal agents by incorporation into nanostructures for drug delivery (6).

A promising alternative can be obtained using nanosystems containing peptide drugs, such as nisin (NIS). This peptide is a bacteriocin produced by Gram-positive 
microorganisms which has bactericidal and bacteriostatic properties, as well as antifungal activity (7). Its effect consists in membrane pore formation of microorganisms (8). Previous reports describe the effectiveness of the NIS against C. albicans strains. The insertion of NIS into drug delivery nanosystems (6) such as liposomes, cyclodextrins, lamellar silicates, dendrimers, nanoemulsions, nanogels, solid lipid nanoparticles, and polymeric nanoparticles $(9,10)$ was already reported in literature.

In order to obtain a new system able to permeate efficiently the most superficial skin layers, forming a drug reservoir with increased stability, the use of insoluble and biodegradable polymeric nanoparticles (NP) arise as one great alternative to enhance the drugs action for the candidiasis control, in particular for treatment of gynecological disorders. Several polymers are used to obtain nanostructured reservoirs, standing out the use of the copolymer of lactic-coglycolic acid-acid (PLGA), poly (cyano acrylate) (PCA), and poly-e-caprolactone (PCL) (11). Under physiological conditions, PCL is degraded by the hydrolysis of ester linkages constituents (12). This degradation mechanism may indicate its potential use in the prepare of nanosystems, which act as reservoir in vaginal mucosa with enhanced mucous penetration in view the preparation of long term implantable devices. Thus, the purpose of this work is the preparation and evaluation of nisin-loaded nanoparticles (NIS-NP), in order to obtain an extended release system for the control of gynecological infections caused by $C$. albicans.

\section{MATERIALS AND METHODS}

\section{Materials}

Purified water was obtained by reverse osmosis and used to prepare culture media, buffers, and solutions and employed in all experiments. Poly- $\varepsilon$-caprolactone (PCL), polyvinyl alcohol (PVA), and nisin 2.5\% were obtained from Sigma-Aldrich (St. Louis, USA-MO). C. albicans strains (ATCC 10231) were provided by INCQS-FIOCRUZ (Rio de Janeiro, Brazil). Dichloromethane was obtained from TEDIA (Rio de Janeiro, Brazil); potato dextrose agar (PDA) culture medium was obtained from DIFCO (Franklin Lakes; USA-NJ) and Pierce ${ }^{\circledR}$ BCA Protein Assay Kit acquired from THERMO SCIENTIFIC (Waltham, USA-MA).

\section{Methods}

\section{Preparation of PCL NIS-Loaded Nanoparticles}

Nanoparticles were prepared using double emulsification method followed by solvent evaporation (13). The first emulsion was obtained by dripping NIS aqueous solution (1 mg/ $\mathrm{mL})$ onto a dichloromethane PCL solution $(2.5 \% \mathrm{w} / \mathrm{v})$ under magnetic stirring, $100 \%$ amplitude of ultrasound probe sonication (UP100H Hielscher Ultrasound), and ice bath. Then, the primary emulsion was dripped onto $40 \mathrm{~mL}$ of $0.1 \%(w / v)$ PVA aqueous solution. The resultant emulsion was sonicated for $5 \mathrm{~min}$. The NIS-NP suspension was obtained after solvent rotary evaporation under reduced pressure. NP were collected by centrifugation at 20,000 rpm for $1 \mathrm{~h}$ and washed three times for non-encapsulated material removal. The supernatant was reserved for NIS loading efficiency assay, while NP pellet was dispersed in $5 \mathrm{~mL}$ of purified water. Blank NP was prepared under the same conditions but without NIS. Process yield was calculated using Eq. 1.

Process Yield $(\%)=\frac{\text { Mass of NP after lyophilization }(\mathrm{mg}) \times 100}{\text { Mass of NIS }(\mathrm{mg})+\text { Mass of PCL }(\mathrm{mg})}$

\section{Determination of NIS Loading Efficiency}

The NIS loading efficiency of the PCL nanoparticles is defined as the NIS loading percentage in relation to the total amount of NIS used during NP preparation. NIS encapsulation was performed by colorimetric assay using BCA protein reagent and detected by UV-VIS (Lambda 35, Perkin Elmer $\left.{ }^{\circledR}\right)$. The BCA protein reagent was added to the sample and incubated at $37^{\circ} \mathrm{C}$ for $30 \mathrm{~min}$. Subsequently, the samples absorbance were measured at $562 \mathrm{~nm}$. Total NIS content inside NIS-NP was determined following complete dissolution of the NIS-NP suspension $(1 \mathrm{~mL})$ in acetonitrile $(20 \mathrm{~mL})$ and water $(20 \mathrm{~mL})$. Methanol $(25 \mu \mathrm{L})$ was then added in order to precipitate the polymer which was eliminated by filtration $(0.45 \mu \mathrm{m})$ and solvents were removed by rotary evaporation under reduced pressure. Free NIS in supernatant was determined also by BCA protein reagent method. A standard curve was obtained by bovine serum albumin (BSA) and NIS in the range concentration of $0-1 \mathrm{mg} / \mathrm{mL}$ (13).

\section{Characterization of Nanoparticle System}

NP were characterized by particle size distribution, zeta potential, surface morphology, Fourier transform infrared (FTIR), differential scanning calorimetry (DSC), and powder $\mathrm{X}$-ray diffraction analysis (XRD). The particle size and polydispersity index of NIS-NP were determined by dynamic light scattering (DLS). In this analysis, NP pellet was dispersed in $10 \mathrm{~mL}$ of distilled water. For zeta potential assessment, the NP suspension was diluted in deionized water and ten measurements were performed for each sample with subsequent calculation of average value (Brookhaven ZetaPALS). FTIR spectra were recorded in the IR Prestige-21 Shimadzu spectrometer, using $1 \% \mathrm{KBr}$ pellets and wavenumber range between 4000 and $400 \mathrm{~cm}^{-1}$. The FTIR spectra were determined for physical mixture of NIS and PCL, blank PCL nanoparticles, and NIS-NP. DSC analysis were performed between 25 and $300^{\circ} \mathrm{C}$ with a Shimadzu DSC-60 thermal analyzer using aluminum pans, which were crimped non-hermetically, with a nitrogen flow of $50 \mathrm{~mL} /$ min and heating rate of $10^{\circ} \mathrm{C} / \mathrm{min}$. Approximately $1 \mathrm{mg}$ of sample was used for DSC analysis, and the instrument was calibrated using indium and zinc Standards. Powder X-ray diffraction patterns were obtained in the Shimadzu XRD 6100, equipped with $\mathrm{Cu}-\mathrm{K} \alpha$ radiation source $(\lambda=1.542 \AA)$, operating at $40 \mathrm{kV}$ and $30 \mathrm{~mA}$ at room temperature.

The diffraction angle $(2 \theta)$ of the patterns was standardized between $2^{\circ}$ and $40^{\circ}(14,15)$. The morphological surface evaluations of blank NP and NIS-NP were performed by scanning electron microscopy (SEM) (JSM 6340, JEOL Datum Ltd.) operating at $3 \mathrm{kV}$ accelerating electron microscope (JSM 6340, JEOL Datum Ltd.) operating at $3 \mathrm{kV}$ accelerating voltage. 
Antifungal Activity

The NIS and NIS-NP antifungal activities were assessed using the pour plate technique and C. albicans strains. Briefly, a small amount of $C$. albicans inoculum $\left(100 \mu \mathrm{L}\right.$ of $10^{4}$ cells/ $\mathrm{mL}), 1 \mathrm{~mL}$ of sample and $20 \mathrm{~mL}$ of PDA medium was added on a Petri dish center. The complete homogenization was carried out using circular movements. The Petri dishes were incubated at $37^{\circ} \mathrm{C}$ for $48 \mathrm{~h}$. A positive control (without sample) and negative (without sample and C. albicans suspension) were also prepared. The minimum inhibitory concentration (MIC) was evaluated by serial dilution of NIS and NIS-NP samples $(16,17)$.

\section{In Vitro NIS Release}

In vitro release experiments were performed using dialysis bag (cellulose acetate membrane; Sigma-Aldrich, St. Louis, USA, MO) inserted in falcon tube containing $30 \mathrm{~mL}$ of biorelevant medium (acetate buffer $\mathrm{pH} 4.0$ ) incubated at $37^{\circ} \mathrm{C}$ in a beaker, containing methyl paraben solution $(0,15 \%$ $w / v)$, to avoid fungal proliferation, with constant shaking (50 rpm). At proper intervals, $3 \mathrm{~mL}$ of medium were sampled and $3 \mathrm{~mL}$ of the fresh solution medium was supplied to keep constant volume. After freeze-drying process, samples were dispersed in $100 \mu \mathrm{L}$ of distilled water and $2.0 \mathrm{~mL}$ of BCA solution were added. The amount of NIS added was determined by the UV/Vis spectrophotometer as previously described. All experiments were performed in triplicate, and the averaged results were obtained. The release profile parameters were calculated by plotting the amount of accumulated release drug against time. The flow through the membrane $(\mathrm{J})$ was calculated from the slopes of the linear portion of the curves, whereas the lag time (tlag) was obtained by extrapolation of the steady-state slope to its intersection on time axis $(18,19)$.

\section{Permeation Experiments}

In vitro permeation experiments were performed using pig vaginal mucosa by Franz diffusion cells. In this assay, the available diffusion area used was $1.767 \mathrm{~cm}^{2}$ and the volume of receptor compartment, containing acetate buffer ( $\mathrm{pH} 4.0)$ at $37^{\circ} \mathrm{C}$, was $7 \mathrm{~mL}$. This volume was sufficient to maintain the sink condition. Samples $(1.0 \mathrm{~mL})$ were taken from the receptor compartment at specified time intervals and immediately lyophilized. After lyophilization, samples were diluted in $100 \mu \mathrm{L}$ of distilled water and $2.0 \mathrm{~mL}$ of BCA were added. Permeation parameters were calculated from the cumulative permeated NIS against time plot. Flow (J) and lag time (tlag) were calculated as described in the previous section (20).

\section{Statistical Analysis}

Comparisons between groups were performed with variance analysis (ANOVA) using the software GraphP. All other statistical analysis was evaluated with this same software.

\section{RESULTS AND DISCUSSIONS}

The most usual encapsulation method for hydrophilic peptides molecules is the double or multiple emulsion technique. The main problem during the development of peptidedrug nanosystems is the low drug yield and the loss of threedimensional structure of these macromolecules, which probably leads to changes of pharmacological or physiological activity related to these bioactive molecules. In this context, the major challenges in the preparation of NIS-NP were to maintain the three-dimensional structure of the peptide and ensure high NIS loading percentages, allowing subsequent relevant scale up strategies. For that reason NIS-NP were prepared at a ratio of 1 part of NIS to 100 parts of polymer. In terms of NP conversion yield, it was shown that almost $60 \%$ of the total polymer mass was converted to NP $(59.38 \pm 0.062945 \%)$, and the average of loading efficiency was $93.88 \pm 3.26 \%(n=10)$. The optimal conditions for preparation described in this work derived from the variation of the concentration of PCL (from 1.0 to $5.0 \% w / v$, at intervals of $0.5 \%$ ) the duration (5 to $10 \mathrm{~min}$ ), and intensity of the ultrasonic energy (60-100\%), and the PVA concentration (from 0.1 to $0.5 \%$, at intervals of $0.1 \%)$. Both the conversion yield of the polymer mass to NISNP as the NIS loading efficiency were considered appropriate for the preparation of NP peptides when compared to the previous reports. In 2011, Soares and co-workers (21) prepared PCL NP containing ZnPc obtained yield of $67.1 \%$. Ossa and co-workers (2012) (22) developed PCL NP containing cannabinoid using the single emulsion method and obtained $58.68 \%$ yield, which was quite similar to the results showed in this study. Similar results of protein loading efficiency (90.6\%) were also observed by De Araújo and co-workers during the development of insulin loaded PCL nanoparticles by doubleemulsion technique (23).

To determine the loading efficiency, a standard curve was prepared with NIS, using the same parameters established by the BCA method for protein detection kit, comparing it with a standard curve of albumin such as recommended by the manufacturer. Both curves showed linear behavior (0.9938 and 0.9967 NIS and albumin, respectively), with no statistical difference between them $(p<0.05)$, confirming the linearity of the method. There was no analysis interference, when tested known amounts of NIS collected with PVA and PCL, confirming the selectivity of the method.

The loading efficiency showed high encapsulation percentage $(93.88 \pm 3.26 \%)$, since practically, the whole peptide was incorporated into the polymer system. Salmaso and coworkers (24) prepared nisin-loaded PLA NP which exhibited $84 \pm 2 \%$ of encapsulation efficiency. Similarly, Prombutara et al. (25) prepared solid lipid NIS nanoparticles obtaining $73.6 \pm 0,04 \%$ of NIS encapsulation. In 2011, Xiao and Zhong (26) also encapsulated NIS into glycerol derivatives and Tween 20 using spray drying technique and obtained an encapsulation efficiency of approximately $50 \%$. Interestingly, the proposed method for development of NIS-NP herein described showed better results than other published studies. This indicates that the methodology developed here may be considered appropriate for the preparation of peptide-loaded $\mathrm{NP}$ with great scale up potential, since it is possible to increase the volume of reaction medium 20 -fold with maintaining of the characteristics shown in the experimental results $(27,28)$. 
According to literature, the optimum particle size range to increase mucoadhesion and retention in vaginal tissue ranges from 200 to $800 \mathrm{~nm}$. Showing size inside this range, nanoparticles can penetrate into human cervicovaginal mucus without expose the drug to systemic circulation. In addition, one particularly important characteristic of any novel delivery system destined for topical administration within the vagina is a low propensity to cause genital irritation and systemic toxicity. Thus, drug delivery strategies that enhance the residence (retention) of antifungal drugs at mucosal tissues while reducing systemic exposure may be interesting toward the development of vaginal microbicides (29-33). Ideally, NIS-NP should have its size adjusted near to $300 \mathrm{~nm}$, in order to enable its penetration into a vaginal mucosa cells, forming a NIS reservoir system, which would be helpful for prevention of C. albicans uncontrolled proliferation (34). In this proposal, size distribution analysis were performed and NIS-NP showed mean diameter of $320.3 \pm 3.38 \mathrm{~nm}$ and polydispersity index (PDI) of $0.21 \pm 0.03$. In order to reduce the mean diameter of NIS-NP, favoring their penetration into the vaginal tissue, the NP production method was adjusted. After the dripping, the NP suspension was subjected to ultrasonic energy for more $5 \mathrm{~min}$ (35). The addition of $5 \mathrm{~min}$ in the adjusted methodology, conducted to smaller particles, $284.8 \pm 5.40 \mathrm{~nm}$ $(\mathrm{PDI}=0.138 \pm 0.03)$ (Fig. 1$)$, makes the system more suitable for application on the vaginal mucosa.

The $\mathrm{pH}$ of NP suspensions presented values around 5.3 for both nanoparticles, blank (PCL NP) and NIS-NP, which is compatible with vaginal mucosa $\mathrm{pH}$. This $\mathrm{pH}$ range may be related to PCL, considering that Ourique and co-workers (36) prepared PCL tretinoin-loaded nanospheres and nanocapsules and measured $\mathrm{pH}$ values ranging from 5.9 to 6.9, as well as, Ribeiro and co-workers (35) which have prepared PCL nanoparticles loaded with Uncaria tomentosa and obtained $\mathrm{pH}$ 5.0, confirming this hypothesis. As can be observed in Fig. 1, zeta potential values for both nanoparticles, PCL NP and NISNP, evidentiate slightly negative nanoparticles, $-6.62 \pm 0.42$ and $-1.96 \pm 0.47$, respectively.

In scanning electronic microscopy (SEM) images (Fig. 1), it is possible to observe that PCL NP shows spherical form, with undefined borders. In addition, the NIS-NP showed a more regular and spherical form when compared to blank

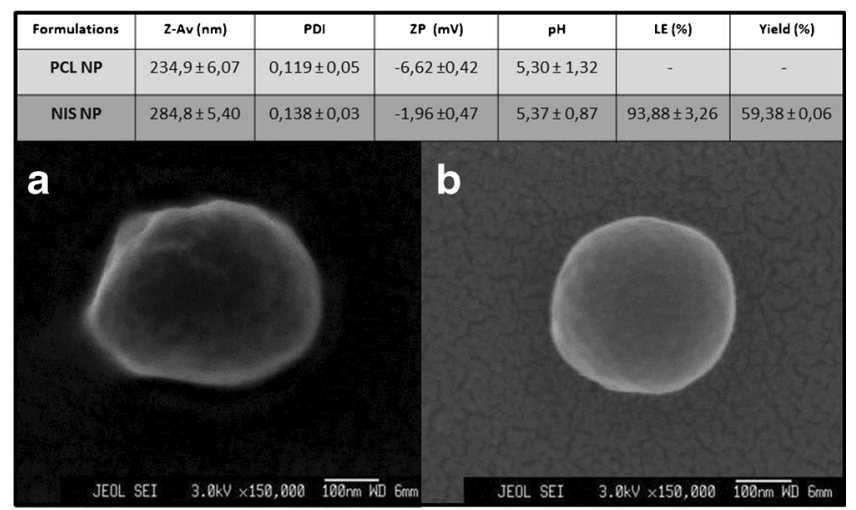

Fig. 1. Characterization of nanoparticles: $Z$-average size $(Z-A v)$, polydispersity index $(P D I)$, zeta potential $(Z P), \mathrm{pH}$, NIS loading efficiency $(L E)$, percentage yield and morphological evaluation by SEM of blank nanoparticles $(P C L N P)(\mathbf{a})$, and nisin-loaded nanoparticles $(N I S-N P)(\mathbf{b})$

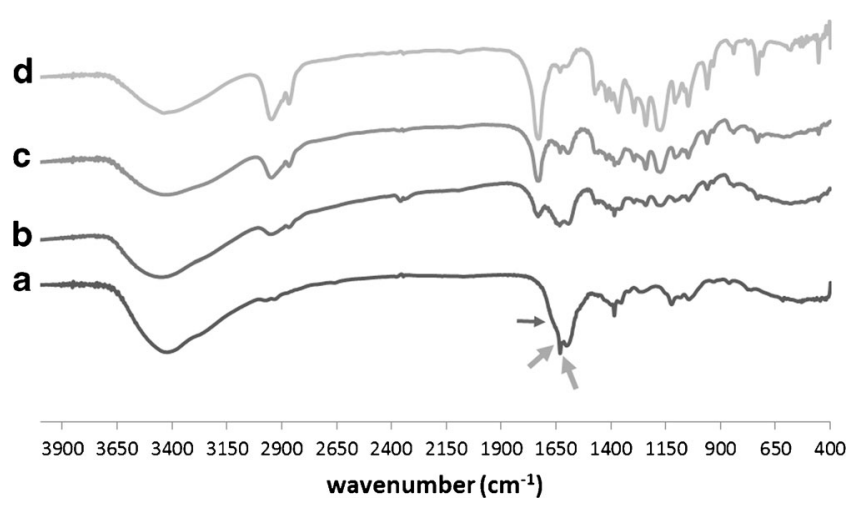

Fig. 2. FTIR spectra of a NIS, b physical mixture, $\mathbf{c}$ blank nanoparticles (PCL NP), and $\mathbf{d}$ nisin-loaded nanoparticles (NIS-NP)

nanoparticles (PCL NP). Both samples did not exhibited polymer or particles agglomeration. The particle size observed by SEM was equivalent to those measured by DLS. Mukerjee and co-workers (37) developed PCL nanoparticles containing albumin, also by double emulsification method using PVA as an emulsifier, and observed NP with spherical form, but it was possible also do observe the presence of pores which was not observed in this study but could indicate an increased mechanical resistance of NIS-NP herein demonstrated (38).

The comparative analysis of FTIR spectra between the physical mixture and NP demonstrates a similar absorption profile; however, physical mixture showed absorption intensities smaller than PCL NP and even smaller than NIS-NP. In 2009, Xue and co-workers (38) have demonstrated the presence of the same characteristic peptide bands by encapsulating albumin in PCL nanoparticles, and also showed that it is possible to observe the maintenance of the secondary structure of proteins by FTIR by decomposition of the amide I bond in fragments by curve fitting method. The band at $1654 \mathrm{~cm}^{-1}$ (black arrow) is attributed to $\alpha$-helix conformation, and the bands at 1628 and $1638 \mathrm{~cm}^{-1}$ (gray arrows) are related to $\beta$-sheet conformation, while the other sub-bands are related to disordered secondary structure, which is observed similarly in NIS and NP samples indicating peptide stabilization $(39,40)$ (Fig. 2).

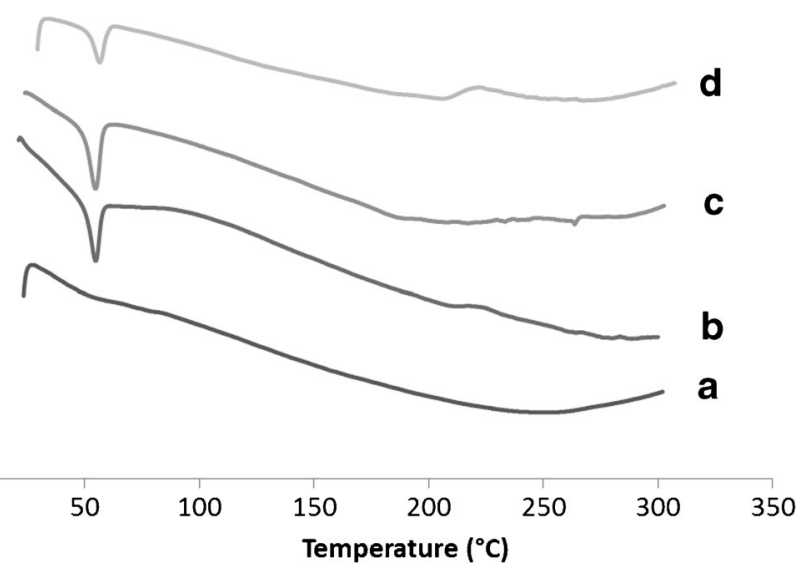

Fig. 3. DSC curves of a NIS, b physical mixture, $\mathbf{c}$ blank nanoparticles (PCL NP), and $\mathbf{d}$ nisin-loaded nanoparticles (NIS-NP) 


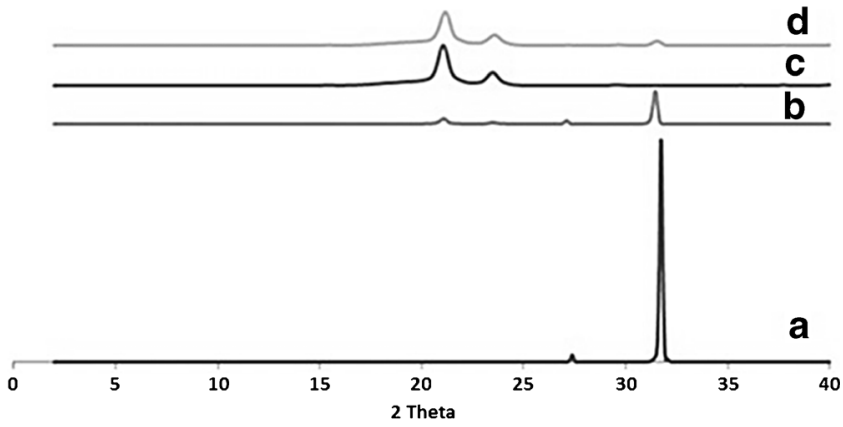

Fig. 4. XRD pattern of a NIS, b nisin-PCL physical mixture, $\mathbf{c}$ blank nanoparticles (PCL NP), and $\mathbf{d}$ nisin-loaded nanoparticles (NIS-NP)

DSC analysis does not provided any indication about possible interactions between NIS and PCL, since the melting point value of the polymer did not show significant variation between curves B, C, and D $\left(54.80,54.82\right.$, and $56.65^{\circ} \mathrm{C}$, respectively) indicating there is no significant interactions with NP components (Fig. 3). However, pure PCL melting point $\left(68.53^{\circ} \mathrm{C}\right)$ decreased after nisin encapsulation $\left(56.65^{\circ} \mathrm{C}\right)$, which confirms the amorphization of the material. It was also observed a lower heat of fusion value $(25.26 \mathrm{~J} / \mathrm{g})$ for nisin PCL nanoparticles than value obtained for pure PCL (111.07 J/g), which indicated a decrease of PCL heat of fusion and consequently its crystallinity.

XRD analysis shows the maintenance of NIS characteristic signal at $32^{\circ}$ ( $2 \theta$ angle) with reduced intensity in the NIS-NP sample, indicating that the peptide is encapsulated into the particle in its crystalline form and consequently without loss of its structural integrity which could indicate the maintenance of its activity. The XRD pattern of blank nanoparticles (PCL-NP) showed two peaks related to semi crystalline polymer structure (41). Nisin-PCL physical mixture XRD pattern showed signals related both to the crystalline structure of the peptide as well the semi crystalline structure of PCL (Fig. 4).

Antifungal activity test was conducted in order to verify if the peptide incorporation into a polymeric nanosystem could affect its antifungal activity. The pour plate technique with cell count was chosen for this evaluation. The freshly diluted peptide and the newly prepared NIS-NP, both at $180 \mu \mathrm{g} / \mathrm{mL}$
Table I. Inhibitory Effect of NP and Nisin on the Growth of C. albicans after 24,48 , and $72 \mathrm{~h}$ of Preparation

\begin{tabular}{llll}
\hline Sample & \multicolumn{3}{l}{ Cell growth $(\%)$} \\
\cline { 2 - 4 } & $24 \mathrm{~h}$ & $48 \mathrm{~h}$ & $72 \mathrm{~h}$ \\
\hline Control & $100 \pm 2.92$ & $100 \pm 2.23$ & $100 \pm 1.94$ \\
NIS-NP & $95.03 \pm 5.43$ & $71.88 \pm 3.51$ & $1.05 \pm 0.99$ \\
NIS & $93.58 \pm 7.48$ & $69.77 \pm 3.75$ & $0.84 \pm 0.88$ \\
\hline
\end{tabular}

of NIS, were added to the petri dish containing inoculum cells. After $24 \mathrm{~h}$ incubation, neither the NIS-NP nor the isolated peptide exhibited considerable antifungal activity. Thus, the antifungal activity was evaluated also at 48 and $72 \mathrm{~h}$ after sample preparation. The results are shown in Table I.

According to the statistical analysis, NIS and NIS-NP samples exhibited statistically similar results in each time $(p<0.05)$. Thus, it is possible to conclude that the NIS activity is not compromised after their loading into PCL nanoparticles. Interestingly, a slight NIS antifungal activity was observed only after $48 \mathrm{~h}$, which could be explained by the fact that NIS is a commercial product and must be released from the formulation $(2.5 \% w / w)$. This happens for peptide commercial formulation (NIS) as well for NISNP formulation.

The pathogenesis of candidiasis can occur through the transition of the blastoconidia form to hyphae form. The efficiency of NIS against infections caused by $C$. albicans was determined by Le Lay and co-workers (6), which observed that NIS Z decreased this transition, although the inhibition was not complete. Posteriorly, Gupta and co-workers (42) investigated the use of NIS as a vaginal contraceptive, due to its spermicidal activity, suggesting also an evaluation of NIS as an antimicrobial agent to prevent vaginal infections. Also in 2009, Akerey and co-workers (43) conducted tests to obtain the MIC of NIS, verifying cell adhesion and strains transfer of the blastoconidia form to hyphae form. The transition was significantly inhibited ( $50 \%$ inhibition) using $5 \mu \mathrm{g} / \mathrm{mL}$ and this effect was dose dependent. These results demonstrated for the first time that NIS, at non-toxic concentrations for human cells, is active against $C$. albicans, suggesting the use of NIS-

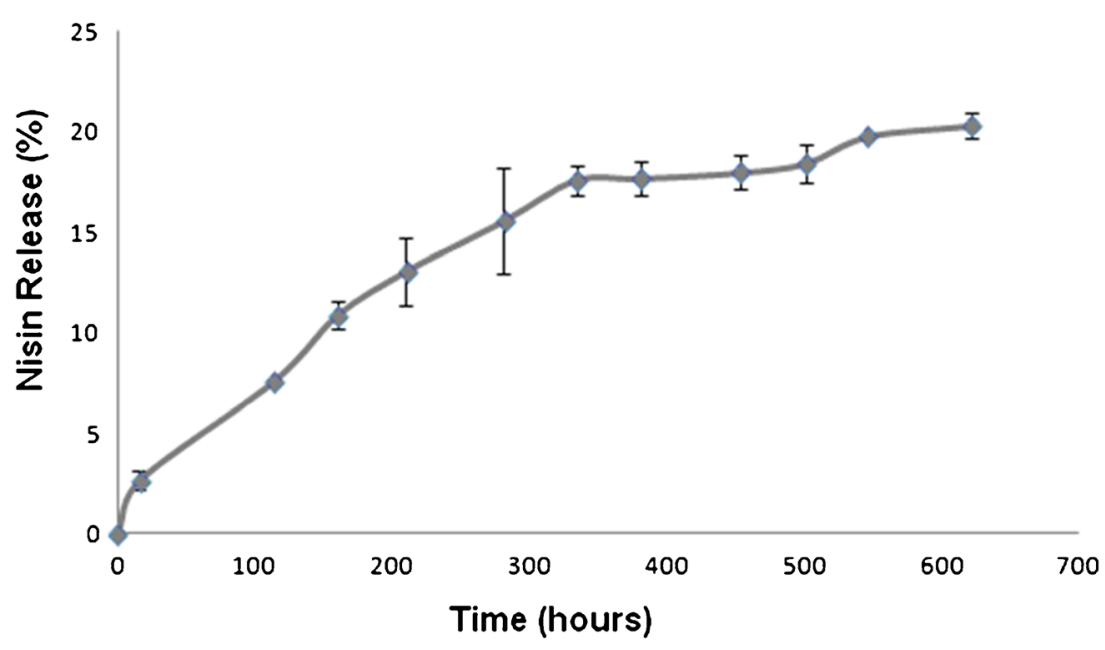

Fig. 5. In vitro release profile (during $621 \mathrm{~h}$ ) of NIS from nisin-loaded nanoparticles in acetate buffer $\mathrm{pH} 4.0,37^{\circ} \mathrm{C}$ 
Table II. NIS-NP and NIS Permeation Parameters After 25 h: Flow Through the Membrane $(J)$, Apparent Permeability Coefficient (Papp) and Lag Time (tlag)

\begin{tabular}{|c|c|c|c|c|}
\hline Formulation $\left(\mu \mathrm{g} / \mathrm{cm}^{2}\right)$ & NIS permeation $\left(\mu \mathrm{g} / \mathrm{cm}^{2} \cdot \mathrm{s}\right)$ & $J \times 10^{-1}$ & Papp $\times 10^{-4}(\mathrm{~cm} / \mathrm{s})$ & tlag (min) \\
\hline NIS-NP & $6.32 \pm 0.46$ & $3.83 \pm 0.58$ & $6.39 \pm 0.97$ & $5.47 \pm 2.90$ \\
\hline NIS & $6.34 \pm 0.85$ & $3.66 \pm 1.89$ & $6.10 \pm 3.14$ & $3.04 \pm 0.58$ \\
\hline
\end{tabular}

NP here prepared as an antifungal agent against gynecological infections.

As can be viewed in Fig. 5, the in vitro release profile of NIS-NP was slow and steady during $621 \mathrm{~h}$. After $16 \mathrm{~h}$, only $2.6 \%$ of NIS were released and after $114 \mathrm{~h}$ (approximately 5 days) almost 7\%. Between days 14 and 20, it was possible to observe an increase in NIS release, reaching almost $18 \%$ of NIS in receptor medium. Only at $621 \mathrm{~h}$, the release percentage has exceeded $20 \%$, showing a sustained and prolonged release profile, like an implantable device.

It is important to notice that during NIS release test, it was observed a release about $5 \%$ after $72 \mathrm{~h}$, in other words, $9.2 \mu \mathrm{g} / \mathrm{mL}$ of NIS in the receptor medium, which is below the MIC $(25 \mu \mathrm{g} / \mathrm{mL})$. In the antifungal activity test (72 h), we observed that NIS antifungal activity was statistically similar to NIS-NP. This delayed antifungal activity could be related to an enzymatic degradation of PCL nanoparticles by enzymes inside $C$. albicans $(12,44,45)$. It is well known that some microorganisms such as Fusarium solani (44) and Streptomyces thermoviolaceus subsp. thermoviolaceus isolate 76T-2 (45) can enzymatically degrade PCL by an esterase, cutinase, also called as PCL depolymerase. These informations about PCL degradation inside fungi and bacteria support the explanation about the behavior of NIS-NP as free peptide, since the concentration of NIS released by the polymer system would be below the MIC.

Different kinetic models were applied on the NIS release values measured to determine the linear correlation coefficient $(r)$. Korsmeyer-Peppas model presented the highest $(r)$ and the best fit to the release profile of nisin-loaded nanoparticles (46). However, the Higuchi model and the zero order also showed satisfactory correlation. According to the applied mathematical models, NIS release occurs by a diffusion mechanism. These results indicate the possibility to use the nanosystem produced as a reservoir with sustained-release mechanism. From the release profile data mathematically determined, the flow through the membrane $(\mathrm{J})$ and the lag time (tlag) based on the kinetics model for formulation were $0.04 \pm$ $0.722 \mathrm{~g} / \mathrm{min}$ and $28.15 \pm 0.03 \mathrm{~h}$, respectively (Table II). The lag time indicates a very high latency, which is a further hint that the developed formulation can be associated with a free NIS attack dose.

The porcine vaginal mucosal permeation (Table II), showed no statistically significant differences between NIS and NIS-NP. Considering the outer layers of the vaginal mucosa as barrier to the permeation test, it is possible to affirm that NP are able to permeate the initial physiological barrier, being deposited in the inner layers of the mucosa, performing the desired reservoir system function for this formulation.

\section{CONCLUSION}

The nanoparticle preparation method developed in this study proved to be appropriated for safe encapsulation of not only NIS but also other peptides, without structural change and activity loss. In this study, we observed a satisfactory yield and high encapsulation efficiency in the proposed nanoencapsulation process. NIS-NP exhibited suitable size, charge, and morphology, being able to permeate vaginal mucosa and act as peptide reservoir system. The test of antifungal activity showed that the NIS encapsulation in a polymer system does not modify the antifungal activity but generated evidences of PCL depolymerization inside C. albicans, since NIS-NP presented very similar behavior than NIS solution. After evaluation of in vitro NIS release and permeation across porcine vaginal mucosa, it was possible to infer that the NIS$\mathrm{NP}$, can act as reservoir system that permeates the initial physiological barriers of the vaginal mucosa and may remain deposited in the inner layers of tissue behaving as a prophylactic agent against candidiasis. Thus, NIS-NP are shown as a potential new therapy system for the treatment of vaginal candidiasis.

\section{ACKNOWLEDGMENTS}

This work was supported by FAPERJ, CNPq e CAPES Edital Nanobiotec 2008.

\section{COMPLIANCE WITH ETHICAL STANDARDS}

Conflict of Interest

competing interests.

The authors declare that they have no

\section{REFERENCES}

1. Alangaden GJ. Nosocomial fungal infections: epidemiology, infection control, and prevention. Infect Dis Clin N Am. 2011;25:201-25.

2. Eggimann P, Garbino J, Pittet D. Epidemiology of Candida species infections in critically ill nonimmunosuppressed patients. Lancet Infect Dis. 2003;3:685-702.

3. Curvelo JAdR, Barreto ALS, Portela MB, Alviano DS, Holandino C, Souto-Padrón T, et al. Effect of the secretory leucocyte proteinase inhibitor (SLPI) on Candida albicans biological processes: a therapeutic alternative? Arch Oral Biol. 2014;59:928-37.

4. de Aguiar MMGB, de Albuquerque RP, Marinho DS, Braga BRS, Dornelas CB, Oliveira A, et al. Oral sustained release nystatin tablets for the treatment of oral candidiasis: formulation development and validation of UV spectrophotometric analytical methodology for content determination. Drug Dev Ind Pharm. 2010;36:594-600.

5. Espinel-Ingroff A. Mechanisms of resistance to antifungal agents: yeasts and filamentous fungi. Rev Iberoam Micol. 2008;25:101-6. 
6. Le Lay C, Akerey B, Fliss I, Subirade M, Rouabhia M. Nisin Z inhibits the growth of Candida albicans and its transition from blastospore to hyphal form. J Appl Microbiol. 2008;105:1630-9.

7. Nascimento M, Finatti D, Moreno I, Kuaye A. Atividade antimicrobiana de Lactococcus lactis subsp. lactis ATCC 11454 produtor de nisina sobre patógenos gram-positivos. Braz J Food Technol. 2008;11:322-8.

8. AlKhatib Z, Lagedroste M, Fey I, Kleinschrodt D, Abts A, Smits SHJ. Lantibiotic immunity: inhibition of nisin mediated pore formation by NisI. PLoS One. 2014;9:e102246.

9. Koo OM, Rubinstein I, Onyuksel H. Role of nanotechnology in targeted drug delivery and imaging: a concise review. Nanomed Nanotechnol Biol Med. 2005;1:193-212.

10. Aguzzi C, Cerezo P, Viseras C, Caramella C. Use of clays as drug delivery systems: possibilities and limitations. Appl Clay Sci. 2007;36:22-36.

11. Mohanraj VJ, Chen Y. Nanoparticles-a review. Trop J Pharm Res. 2007;5:561-73.

12. Fukushima K, Abbate C, Tabuani D, Gennari M, Rizzarelli P, Camino G. Biodegradation trend of poly ( $\varepsilon$-caprolactone) and nanocomposites. Mater Sci Eng C. 2010;30:566-74.

13. Jelvehgari M, Barar J, Valizadeh H, Heidari N. Preparation and evaluation of poly ( $\epsilon$-caprolactone) nanoparticles-inmicroparticles by W/O/W emulsion method. Iran J Basic Med Sci. 2010; 13.

14. Zabska M, Jaskiewicz K, Kiersnowski A, Szustakiewicz K, Rathgeber S, Piglowski J. Spontaneous exfoliation and selfassembly phenomena in polyvinylpyrrolidone/synthetic layered silicate nanocomposites. Radiat Phys Chem. 2011;80:1125-8.

15. Koo J. Polymer nanocomposites: processing, characterization, and applications. 1st ed. New York: McGraw-Hill Professional; 2006.

16. Pupe CG, Villardi M, Rodrigues CR, Rocha HVA, Maia LC, de Sousa VP, et al. Preparation and evaluation of antimicrobial activity of nanosystems for the control of oral pathogens Streptococcus mutans and Candida albicans. Int $\mathrm{J}$ Nanomedicine. 2011;6:2581.

17. Mosca DA, Hurst MA, So W, Viajar BS, Fujii CA, Falla TJ. IB367 , a protegrin peptide with in vitro and in vivo activities against the microflora associated with oral mucositis. Antimicrob Agents Chemother. 2000;44:1803-8.

18. Sathler PC, Lourenço AL, Rodrigues CR, da Silva LCRP, Cabral $\mathrm{LM}$, Jordão $\mathrm{AK}$, et al. In vitro and in vivo analysis of the antithrombotic and toxicological profile of new antiplatelets $\mathrm{N}$ acylhydrazone derivatives and development of nanosystems. Thromb Res. 2014;134:376-83.

19. Modi S, Anderson BD. Determination of drug release kinetics from nanoparticles: overcoming pitfalls of the dynamic dialysis method. Mol Pharm. 2013;10:3076-89.

20. Moser K, Kriwet K, Naik A, Kalia YN, Guy RH. Passive skin penetration enhancement and its quantification in vitro. Eur $\mathbf{J}$ Pharm Biopharm. 2001;52:103-12.

21. da Volta Soares M, Oliveira MR, Dos Santos EP, de Brito Gitirana L, Barbosa GM, Quaresma CH, et al. Nanostructured delivery system for zinc phthalocyanine: preparation, characterization, and phototoxicity study against human lung adenocarcinoma A549 cells. Int J Nanomed. 2011;6:227-38.

22. de la Ossa DHP, Ligresti A, Gil-Alegre ME, Aberturas MR, Molpeceres J, Di Marzo V, et al. Poly-e-caprolactone microspheres as a drug delivery system for cannabinoid administration: Development, characterization and in vitro evaluation of their antitumoral efficacy. J Control Release. 2012;161:927-32.

23. de Araujo TM, Teixeira Z, Barbosa-Sampaio HC, Rezende LF, Boschero AC, Duran $\mathrm{N}$, et al. Insulin-loaded poly ( $\varepsilon-$ Caprolactone) nanoparticles: efficient, sustained and safe insulin delivery system. J Biomed Nanotechnol. 2013;9:1098-106.

24. Salmaso S, Elvassore N, Bertucco A, Lante A, Caliceti P. Nisinloaded poly-L-lactide nanoparticles produced by CO 2 antisolvent precipitation for sustained antimicrobial activity. Int J Pharm. 2004;287:163-73.

25. Prombutara P, Kulwatthanasal Y, Supaka N, Sramala I, Chareonpornwattana S. Production of nisin-loaded solid lipid nanoparticles for sustained antimicrobial activity. Food Control. 2012;24:184-90.

26. Xiao $\mathrm{D}$, Zhong $\mathrm{Q}$. In vitro release kinetics of nisin as affected by Tween 20 and glycerol coencapsulated in spray-dried zein capsules. J Food Eng. 2011;106:65-73.

27. Guerreiro LH, Da Silva D, Ricci-Junior E, Girard-Dias W, Mascarenhas CM, Sola-Penna M, et al. Polymeric particles for the controlled release of human amylin. Colloids Surf B: Biointerfaces. 2012;94:101-6.

28. Erdemli Ö, Usanmaz A, Keskin D, Tezcaner A. Characteristics and release profiles of MPEG-PCL-MPEG microspheres containing immunoglobulin G. Colloids Surf B: Biointerfaces. 2014:117:487-96.

29. Yang M, Lai SK, Yu T, Wang Y-Y, Happe C, Zhong W, et al. Nanoparticle penetration of human cervicovaginal mucus: the effect of polyvinyl alcohol. J Control Release Off J Control Release Soc. 2014;192:202-8.

30. Vanić Ž, Škalko-Basnet N. Nanopharmaceuticals for improved topical vaginal therapy: can they deliver? Eur J Pharm Sci Off J Eur Fed Pharm Sci. 2013;50:29-41.

31. das Neves J, Araújo F, Andrade F, Michiels J, Ariën KK, Vanham $\mathrm{G}$, et al. In vitro and ex vivo evaluation of polymeric nanoparticles for vaginal and rectal delivery of the anti-HIV drug dapivirine. Mol Pharm. 2013;10:2793-807.

32. das Neves J, Amiji M, Sarmento B. Mucoadhesive nanosystems for vaginal microbicide development: friend or foe? Wiley Interdiscip Rev Nanomed Nanobiotechnol. 2011;3:389-99.

33. Lai SK, Wang Y-Y, Hida K, Cone R, Hanes J. Nanoparticles reveal that human cervicovaginal mucus is riddled with pores larger than viruses. Proc Natl Acad Sci U S A. 2010;107:598-603.

34. Yoo J-W, Giri N, Lee CH. pH-sensitive Eudragit nanoparticles for mucosal drug delivery. Int J Pharm. 2011;403:262-7.

35. Ribeiro AF, de Oliveira Rezende RL, Cabral LM, de Sousa VP. Poly $\varepsilon$-caprolactone nanoparticles loaded with Uncaria tomentosa extract: preparation, characterization, and optimization using the Box-Behnken design. Int $\mathrm{J}$ Nanomedicine. 2013;8:431.

36. Ourique AF, Pohlmann AR, Guterres SS, Beck RCR. Tretinoinloaded nanocapsules: preparation, physicochemical characterization, and photostability study. Int J Pharm. 2008;352:1-4.

37. Mukerjee A, Sinha VR, Pruthi V. Preparation and characterization of poly-e-caprolactone particles for controlled insulin delivery. J Biomed Pharm Eng. 2007;1:40-4.

38. Xue W, Bandyopadhyay A, Bose S. Polycaprolactone coated porous tricalcium phosphate scaffolds for controlled release of protein for tissue engineering. J Biomed Mater Res B Appl Biomater. 2009;91:831-8.

39. Kong J, Yu S. Fourier transform infrared spectroscopic analysis of protein secondary structures. Acta Biochim Biophys Sin. 2007;39:549-59.

40. Barth A. Infrared spectroscopy of proteins. Biochim Biophys Acta Bioenerg. 2007;1767:1073-101.

41. Gautam S, Dinda AK, Mishra NC. Fabrication and characterization of PCL/gelatin composite nanofibrous scaffold for tissue engineering applications by electrospinning method. Mater Sci Eng C. 2013;33:1228-35.

42. Gupta SM, Aranha CC, Bellare JR, Reddy KVR. Interaction of contraceptive antimicrobial peptide nisin with target cell membranes: implications for use as vaginal microbicide. Contraception. 2009;80:299-307.

43. Akerey B, Le-Lay C, Fliss I, Subirade M, Rouabhia M. In vitro efficacy of nisin $\mathrm{Z}$ against Candida albicans adhesion and transition following contact with normal human gingival cells. J Appl Microbiol. 2009;107:1298-307.

44. Murphy CA, Cameron JA, Huang SJ, Vinopal RT. Fusarium polycaprolactone depolymerase is cutinase. Appl Environ Microbiol. 1996;62:456-60.

45. Chua T-K, Tseng M, Yang M-K. Degradation of Poly ( $\varepsilon-$ caprolactone) by thermophilic Streptomyces thermoviolaceus subsp. thermoviolaceus 76T-2. AMB Expr. 2013;3:8.

46. Dash S, Murthy PN, Nath L, Chowdhury P. Kinetic modeling on drug release from controlled drug delivery systems. Acta Pol Pharm. 2010;67:217-23. 